\title{
The clinical oro-facial findings of an 11-year-old Japanese boy with 47, XYY: A case report
}

\author{
Sachiko Hayashi-Sakai*1, Jun Sakai*2, Takuya Kitamura*3, \\ Makoto Sakamoto*3 and Yo Taguchi*1 \\ *1 Division of Pediatric Dentistry, Department of Oral Health Science, Course for Oral Life Science, \\ Niigata University Graduate School of Medical and Dental Sciences \\ 2-5274 Gakkocho-dori, Chuo-ku, Niigata 951-8514, JAPAN \\ *2 Department of System and Automotive Engineering, Niigata College of Technology \\ 5-13-7 Kamisinei-cho, Nishi-ku, Niigata 950-2076, JAPAN \\ *3 Department of Basic Radiological Technology, Niigata University School of Health Sciences, Faculty of Medicine \\ 2-746 Asahimachi-dori, Chuo-ku, Niigata 951-8518, JAPAN
}

\begin{abstract}
XYY is a sex chromosome-number disorder occurring in males. Pediatric dentists seldom report encountering 47, XYY males, probably because there are no overt signs of the disease. The purpose of this report is to present the clinical oro-facial findings of the case of a 47, XYY boy based on cephalometric analysis and study model examinations as well as micro-CT measurement of tooth mineralization. An 11-year-old Japanese boy was referred to our clinic for extraction of primary teeth because of their prolonged retention. He had 47, XYY disorder, pulmonary hypertension, and slight mental retardation. Cephalometric analysis and study model examinations that included a comparison of tooth size and arch assessments were performed. A further attempt was made to demonstrate the degree of dentin mineralization in primary molars. The boy's height and weight were normal but his head circumference had exceeded the 94th percentile since 2 years of age. The cephalometric analysis showed an increase in the lower facial height and bimaxillary protrusion with a longer mandible. Study model examination revealed that the mesio-distal crown widths of all erupted primary and permanent teeth were larger than the standard values, except for the mandibular permanent canines. The degree of dentin mineralization was almost normal in the crown and root areas. A profilogram showed an increase in the lower facial height and bimaxillary protrusion with a longer mandible. The dental arch showed a remarkably contracted width but an extended length. The crown width was larger than the standard values, but the distribution of the degree of dentin mineralization differed little from normal.
\end{abstract}

Key words 47, XYY males, Cephalometric analysis, Dental arch, Dental mineralization degree, Tooth size

\section{Introduction}

47, XYY is one of several sex chromosomenumeral disorders, and exclusively affects males. This chromosomal constitution was first observed by Sandberg et al. in $1961^{1)}$. When the disorder was first discovered in adults, studies of 47, XYY individuals created a stereotype of affected individuals

Received on December 10, 2007

Accepted on June 16, 2008 as exhibiting deviant behavior marked by physical aggressiveness and violence. It now appears that adults with this karyotype may be relatively impulsive, antisocial, and likely to break the law, but they are not especially aggressive. Approximately 1 of 1,000 newborn males has an XYY chromosome pattern $^{2,3}$. In the infancy, the $47, \mathrm{XYY}$ males show normal birth weight, height and head circumference, but in adulthood, tall stature is characteristic. Indeed, almost all body, head, and cephalometric dimensions of 47, XYY adults are larger than those 

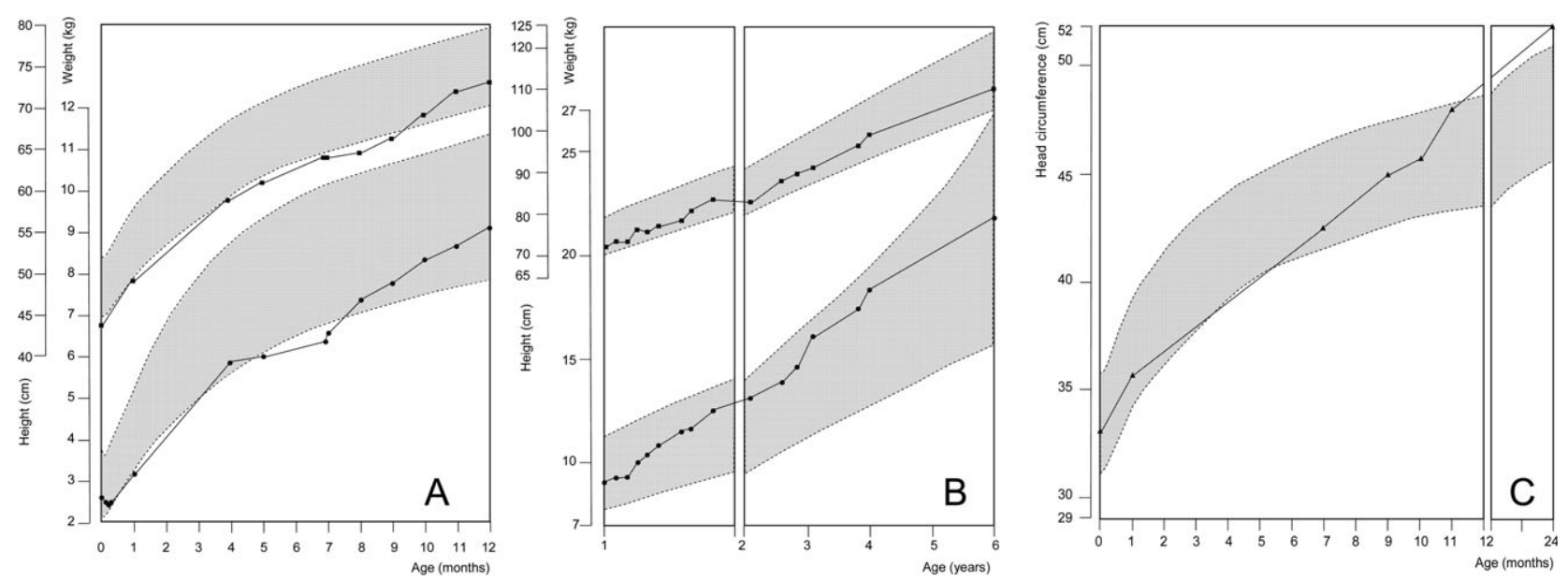

Fig. 1 Growth curves in infancy and early childhood

(A) Height (circles) and weight (squares) in infancy. (B) Height and weight in early childhood. (C) Head circumference (triangles) in infancy and early childhood. The shaded zones represent the 94th percentile in Japanese boy.

in normal males with similar body proportions $\mathrm{s}^{4,5}$.

It has been reported that the tooth size in 47 , $\mathrm{XYY}$ males is larger than that of normal males in both primary and permanent dentitions ${ }^{3,6,7)}$. However, little information is available regarding 47, XYY boys with mixed dentition because these children have no signs of disease and thus are seldom identified.

The aim of the present report is to describe clinical oro-facial findings of an 11-year-old 47, XYY boy based on cephalometric analysis and an examination of study models that includes a comparison of the primary and permanent teeth sizes and arch assessments. A further attempt has been made to demonstrate the degree of dentin mineralization of the primary molars.

\section{Case Report}

An 11-year-old Japanese boy was referred to the Pediatric Dental Clinic of Niigata University Medical and Dental Hospital from a private dental clinic for the extraction of maxillary primary first molars and a mandibular right canine due to their prolonged retention.

The patient's intranatal data were collected. He was a term infant delivered normally at 39 weeks of pregnancy. His birth weight was $2,622 \mathrm{~g}$, his height $43.9 \mathrm{~cm}$, his chest circumference $30.2 \mathrm{~cm}$, and his head circumference $33.0 \mathrm{~cm}$, all of which were within normal range. His growth curve was almost normal except for his head circumference, which

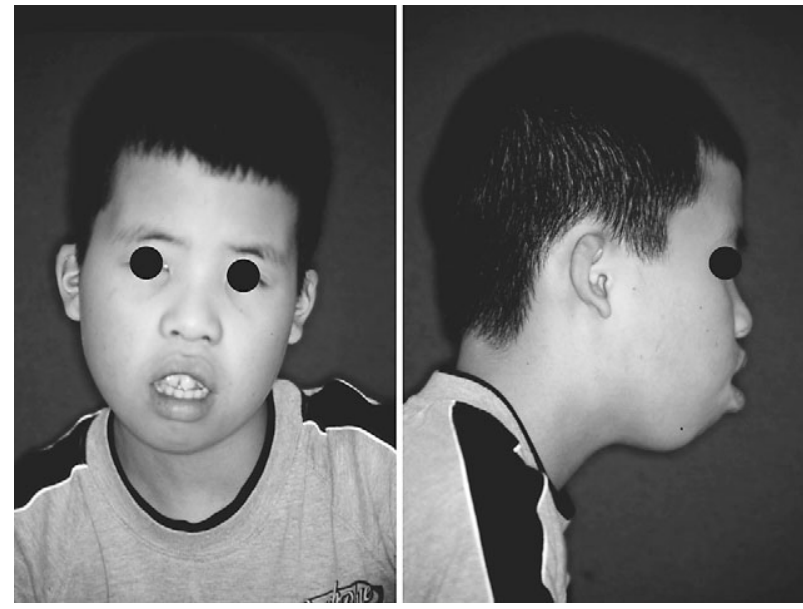

Fig. 2 Clinical extra-oral views at 11 years of age

exceeded 94th percentile from 2 years of age (Fig. $1 \mathrm{~A}-\mathrm{C}$ ). At 11 years of age, when he first visited our hospital, his weight was $39 \mathrm{~kg}$ (average for his age group: $39.1 \pm 9.16 \mathrm{~kg}$ ) and his height was $145 \mathrm{~cm}$ (average for his age group: $145.1 \pm 7.14 \mathrm{~cm}$ ). On the other hand, the cephalometric radiograph examination showed that his head circumference remained large, as described below.

The patient underwent surgery for heart disease with an atrial septal defect at the age of 6 months. In addition, he had pulmonary hypertension and slight mental retardation. After contacting his pediatrician, it was found that he was also a $47, \mathrm{XYY}$ male. Haloperidol and biperiden hydrochloride were prescribed to school-related stress. There were no 


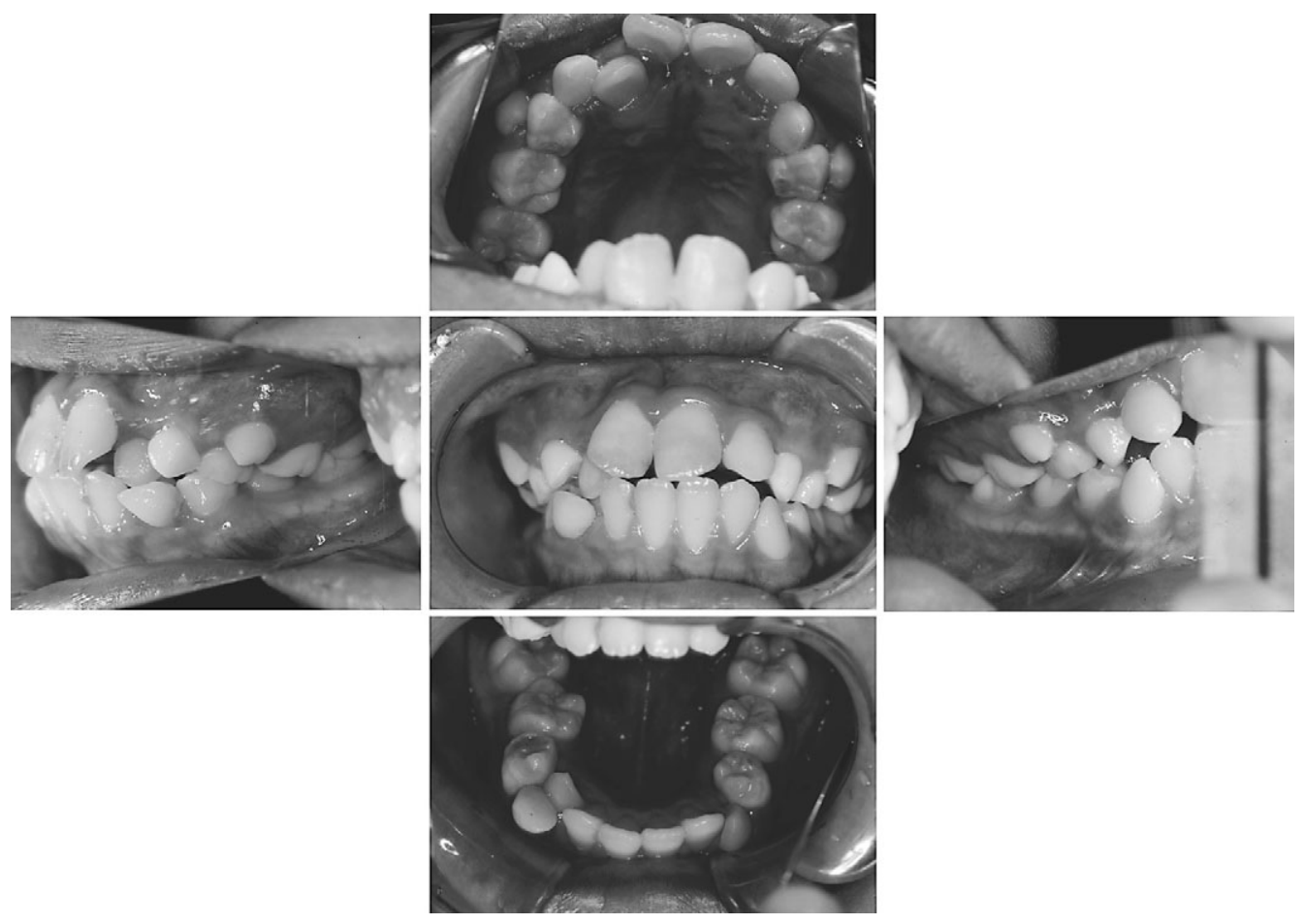

Fig. 3 Clinical intra-oral views at 11 years of age
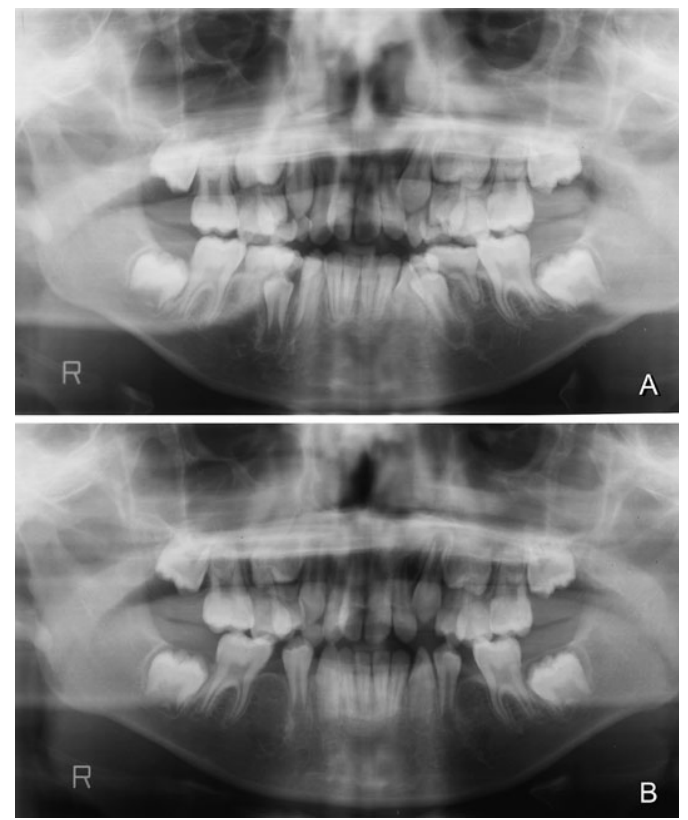

Fig. 4 Radiographic appearance at (A) 11 years and (B) 11 years 5 months

relevant conditions and events in the medical history of his family members.

Extra-oral findings revealed the presence of mild facial asymmetry and glabellar mounding (Fig. 2).

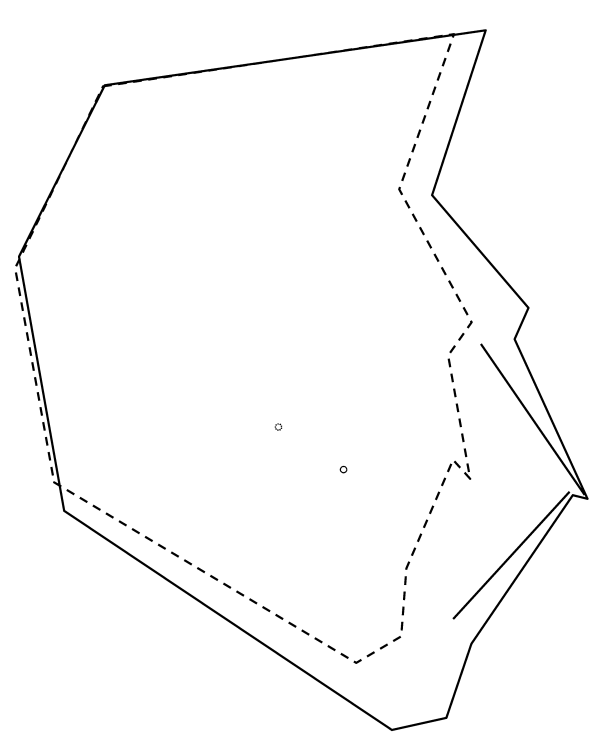

Fig. 5 Profilogram from the cephalometric radiograph analysis

The solid line represents the present case, and the broken line normal standard values.

The clinical examination showed that all erupted teeth were caries-free (Fig. 3). Radiographical findings revealed that both mandibular second premolars were congenitally missing (Fig. 4A). He 

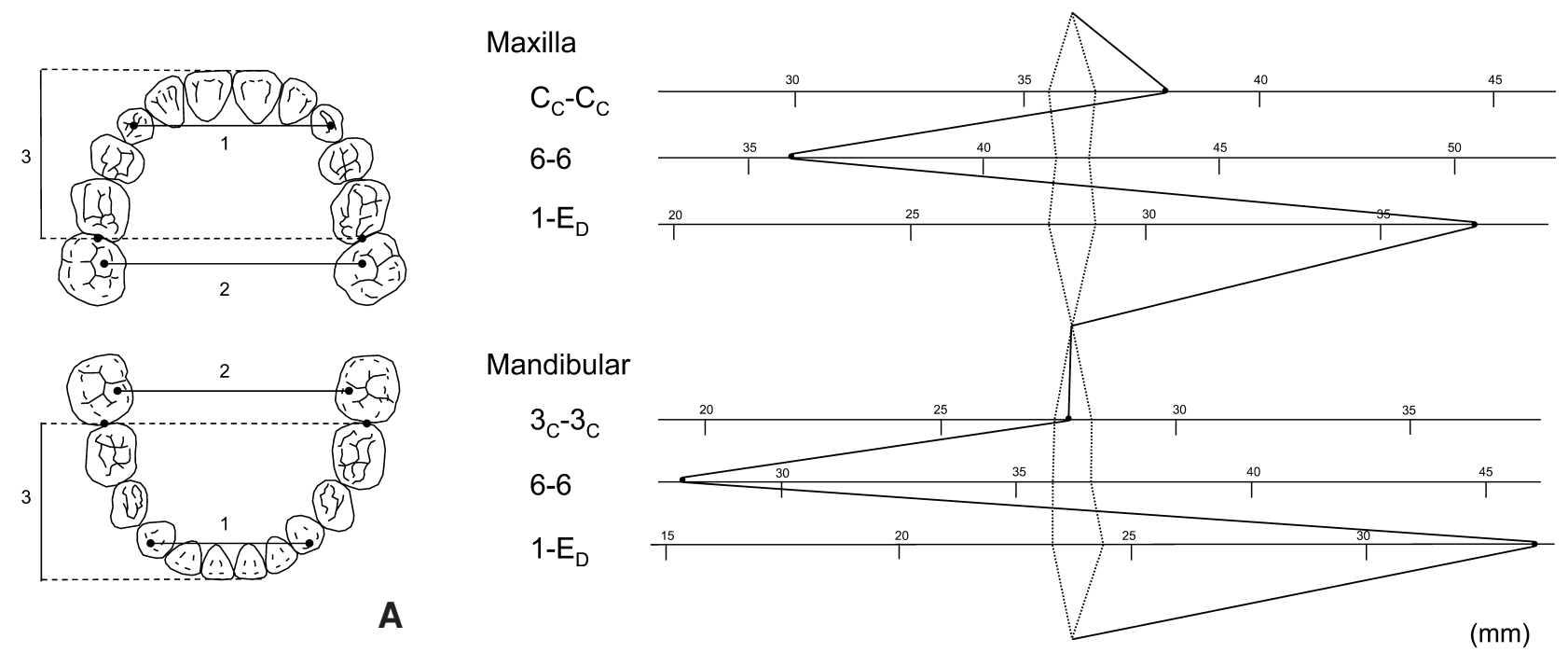

A

Fig. 6 (A) Inspection items for the dental arch width and length in the study model examination. 1: Inter-cusp of primary canines in the maxilla $\left(\mathrm{C}_{\mathrm{C}}-\mathrm{C}_{\mathrm{C}}\right)$ and permanent canines in the mandible $\left(3_{\mathrm{C}}-3_{\mathrm{C}}\right) .2$ : Inter-buccal groove of permanent first molars (6-6). 3: Inter-labial surfaces of permanent central incisors and the distal surfaces of primary second molars (1- $\mathrm{E}_{\mathrm{D}}$ ).

(B) Comparison with normal standard values (broken line)

had no oral habits, abnormal tongue position, or mouth-breathing.

\section{Treatment}

Both maxillary primary first molars and the mandibular right canine were extracted due to prolonged retention. The mandibular primary second molars with severe resorption of the mesial roots were also extracted, due to their poor retention and because their successors were congenitally missing. After 5 months, the mandibular first molars moved toward the mesial and the extracted spaces closed almost spontaneously (Fig. 4B).

\section{Cephalometric radiograph analysis}

For the cephalometric landmarks and standard values, data from the Japanese Society of Pediatric Dentistry were used ${ }^{8)}$. All subjects in the database had normal occlusion and were aged from 8 years 11 months to 13 years (mean age: 11 years old). The profilogram was compared with standard values to display the differences in skeletal features (Fig. 5). This comparison showed an increase in the S-N length and a lower facial height as well as bimaxillary protrusion with a longer mandible.

\section{Study model examination}

1) Dental arch width and length

The dental arch width and length were measured using a sliding caliper with an accuracy of $0.05 \mathrm{~mm}$. The landmarks used in the present study were defined by Moorrees ${ }^{9}$. The dental arch was measured according to Otsubo's method for cases of mixed dentition ${ }^{10}$. The arch width and length were measured and compared with standard values in Japanese children with normal occlusion at the same age; the inspection items considered included the mandibular inter-cusp width of permanent canines $\left(3_{\mathrm{C}}-3_{\mathrm{C}}\right)$, maxillary inter-cusp width of primary canines $\left(\mathrm{C}_{\mathrm{C}}-\mathrm{C}_{\mathrm{C}}\right)$, maxillary and mandibular inter-buccal groove width of first molars (6-6), and the arch length between the inter-labial surfaces of the permanent central incisors and the distal surfaces of primary second molars $\left(1-\mathrm{E}_{\mathrm{D}}\right)$ (Fig. 6A) ${ }^{10)}$. Although $33_{\mathrm{C}}-3_{\mathrm{C}}$ was within the normal range, $\mathrm{C}_{\mathrm{C}^{-}} \mathrm{C}_{\mathrm{C}}$ and $1-\mathrm{E}_{\mathrm{D}}$ exceeded the mean plus the standard deviation, and maxillary and mandibular 6-6 were quite small, far from the mean value (Fig. 6B). As a result, both the maxilla and mandible showed a remarkably contracted dental arch in the molar region, but an extended dental arch length.

2) Mesio-distal crown widths

The mesio-distal crown widths of primary and permanent teeth crowns were measured according to the method and standard values defined by Otsubo and the Japanese Society of Pediatric Dentistry ${ }^{11,12)}$. The mesio-distal crown widths of all erupted primary 


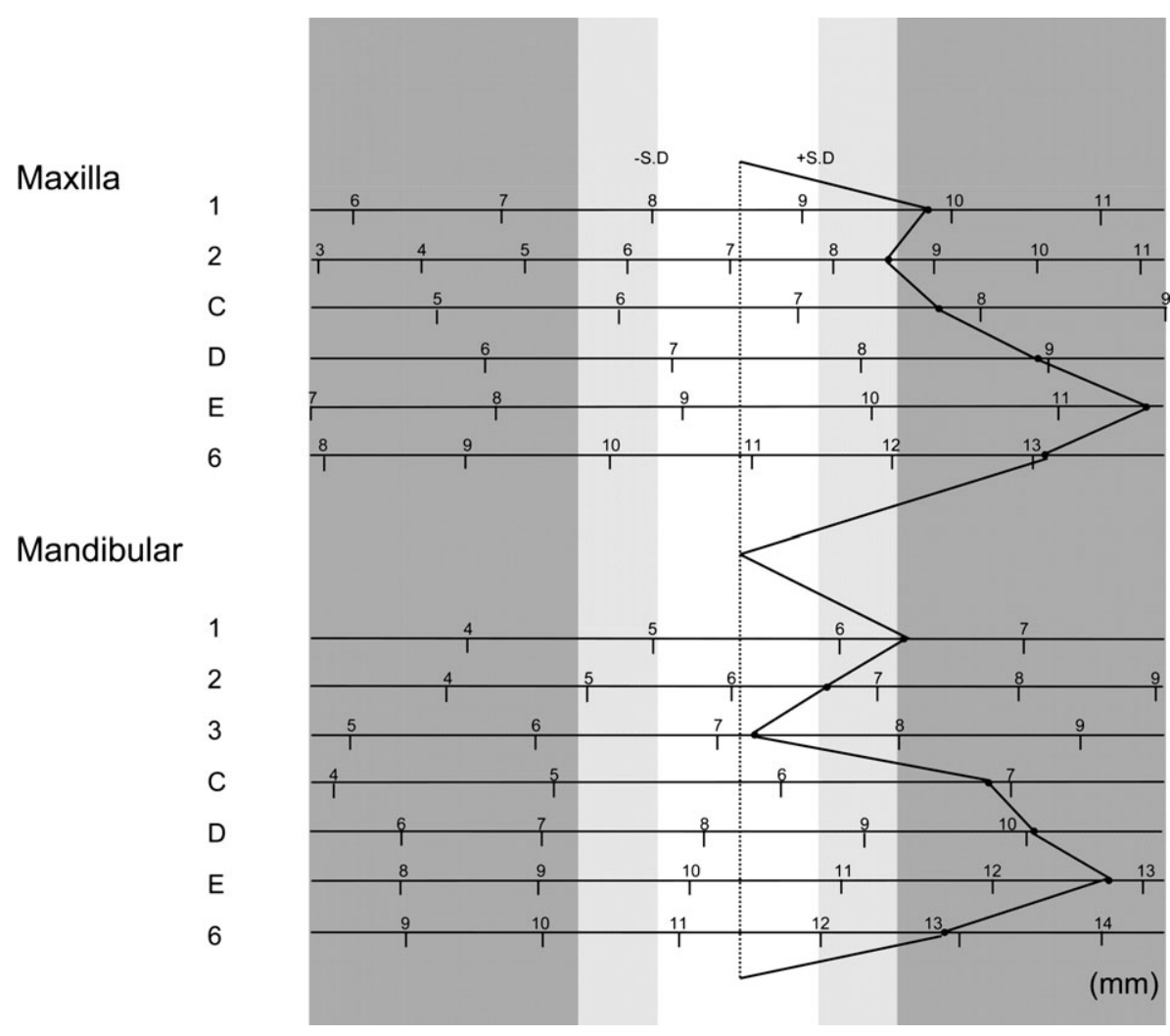

Fig. 7 Mesio-distal crown widths are shown as a comparison with normal standard values

and permanent teeth were larger than the standard values in normal Japanese children, except for those of mandibular permanent canines (Fig. 7). The following tendencies were noted:

1. The values of the primary molars were further away from the standards than those of the permanent teeth.

2. The values of the posterior teeth were more deviated from normal than those of the anterior teeth. Therefore, the maxillary and mandibular primary second molars were markedly larger than standard values.

\section{Degree of dentin mineralization in primary molars}

The three-dimensional geometry of both extracted mandibular second primary molars for which the distal roots were not physiological resorbed were analyzed. For purpose of comparison, sound mandibular primary second molars that were extracted from a normal boy for tooth alignment at 12 years 2 months of age were used as a control. These samples were analyzed using a micro-CT (SMX-130CT-SV; Shimadzu Co., Tokyo, Japan). For measurement of the degree of dentin mineralization in primary molars, a degree of mineralization of bone (DMB) quantitative evaluation system was applied to the investigation. This system is a simple, accurate and novel mineralization measuring system ${ }^{13,14)}$.

To determine the degree of dentin mineralization, a phantom containing $\mathrm{K}_{2} \mathrm{HPO}_{4}$ solutions of known concentrations was developed. Since there was a linear relationship between the CT number and the concentration of the $\mathrm{K}_{2} \mathrm{HPO}_{4}$ solutions, the degree of dentin mineralization could be estimated from the CT numbers of the $\mathrm{K}_{2} \mathrm{HPO}_{4}$ solution in the phantom using the least squares method. Extracted primary teeth were examined and analyzed as 300 cross-sectioned slices on the micro-CT. The resolution of the micro-CT images was confirmed to be $58 \mu \mathrm{m} /$ pixel. In the present study, each micro-CT image was picked up and observed. Figure 8 shows a micro-CT image of the primary second molar in a cross-section of the crown area. There were few differences in the distribution of the degree of dentin mineralization between our patient with 47, XYY and the normal boy in the cross-section of the crown area. The comparison of the degree of 


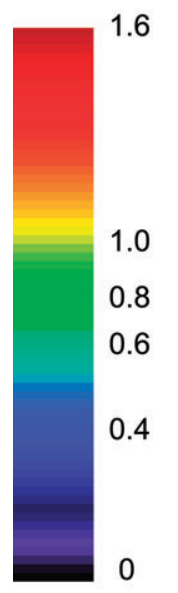

$\left(\mathrm{g} / \mathrm{cm}^{3}\right)$
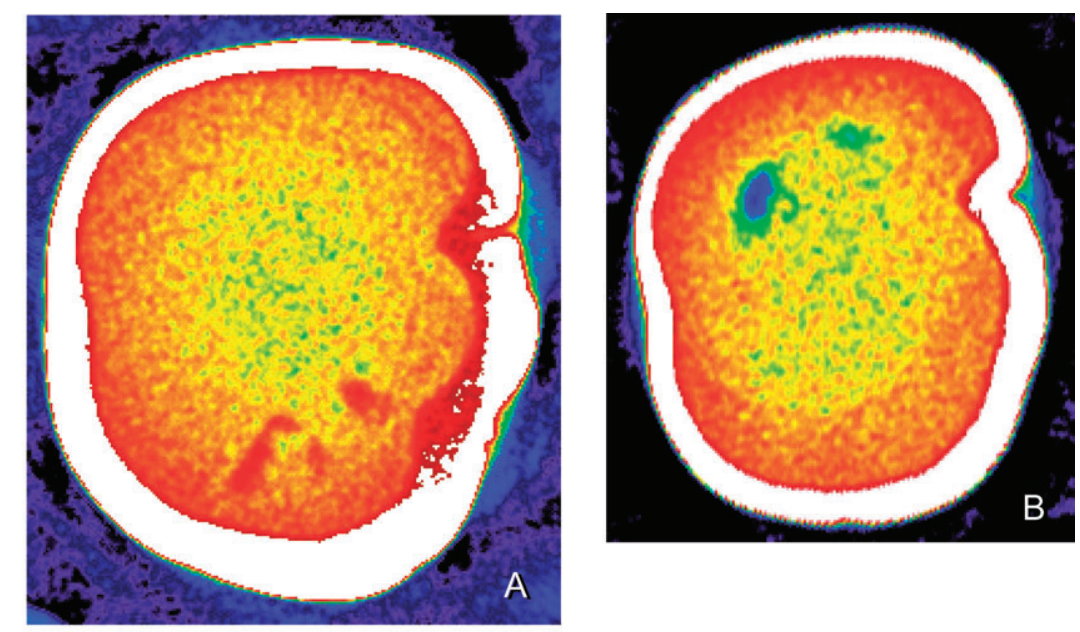

Fig. 8 Micro-CT images of primary molars of the present case (A) and a control (B) in a cross-section of the crown area

Both images have the same magnification ratio.

dentin mineralization in the root area also showed no relevant difference between the patient and the control.

\section{Discussion}

\section{General conditions and anomalies}

In the present case, the birth weight, height, chest circumference, and head circumference were within the 94th percentile on the normal growth curve, but the head circumference was beyond the normal growth curve from 2 years of age. At the age of 11 years, although the patient's height was still normal, his profilogram showed an increase in the S-N length and lower facial height, as well as bimaxillary protrusion with a longer mandible. It has been reported that 47, XYY males do not differ from normal infants in birth weight, stature, and head circumference, but that the velocity of height growth increases significantly from 2 years of age ${ }^{15}$. By 5 years of age, boys with 47, XYY are above the 50th percentile for height and approximately 38\% are above the 90th percentile. Grön et al. have stated that 47, XYY adult males exhibit an increased lower facial height with posterior rotation of the mandible; they also show a tendency to bimaxillary protrusion with a longer mandible and a smaller ramus inclination ${ }^{5}$. The findings in the present case are in approximate agreement with these previously reported adult tendencies, except with respect to the height growth.
It has been reported that approximately $20 \%$ of the patients have various minor anomalies ${ }^{3}$. The observed minor anomalies and subtle phenotypic alterations included mild facial asymmetry, glabellar mounding, long ears, a highly arched palate, and a bony chin point ${ }^{3)}$. There has also been a case report of a 47, XYY patient with double lip, cleft palate, skeletal mandibular protrusion with congenitally missing teeth, and velopharyngeal incompetence ${ }^{16)}$. The present case had mild facial asymmetry, glabellar mounding and both mandibular second premolars were congenitally missing, but the other previously reported anomalies were not observed.

\section{Dental anomalies}

According to our survey of the literature, there have been only 4 case reports on 47 , XYY males over the last 2 decades in the oral and maxillofacial field ${ }^{16)}$. In addition, there have been no reports that include a comparison of primary and permanent teeth sizes during mixed dentition in the same individual, or an analysis of dentin mineralization.

In the present case, the mesio-distal crown widths in primary teeth were remarkably larger than those of normal boys, and the crown widths of the erupted permanent teeth were also larger, except for those of the mandibular canines. Several reports have indicated that both primary and permanent teeth dimensions in 47, XYY males tend to be larger than those of normal males ${ }^{6,7)}$. Townsend et al. ${ }^{7)}$ have noted that the crown sizes of affected males 
are significantly larger on the primary canines and molars, and that permanent teeth dimensions are also larger except for the maxillary permanent canine. Their findings roughly correspond to those in the present case.

Based on their findings, Townsend et al. ${ }^{7)}$ have hypothesized that the earlier-forming permanent teeth tend to be more affected and larger in dimension than the later-forming ones. This trend could not be fully applied to the present case of mixed dentition, since the primary second molars exhibited the largest width among the primary teeth. Our results therefore suggest that the size of the primary molars is more affected than that of the permanent molars, and that the posterior teeth are more deviated in dimension from the normal than the anterior teeth. Therefore, maxillary and mandibular primary second molars would be remarkably large in comparison with standard values. It has been hypothesized that the $\mathrm{Y}$ chromosome affects tooth crown growth in normal males ${ }^{7)}$. Therefore, it may be that the extra Y chromosome in 47, XYY males is responsible for the increase in size of the primary and/or posterior teeth.

As a result of the examination of study models, it was revealed that both the maxilla and mandible showed a remarkably contracted dental arch in the molar region, but an extended dental arch length. These findings have not been previously reported but might be related to the present syndrome as minor anomalies.

As for the distribution of the degree of dentin mineralization on mandibular molars, there were few differences in distribution between our patient with 47, XYY and the normal boy used for comparison. It was indicated that the $47, \mathrm{XYY}$ boy had a large tooth size with an almost normal degree of dentin mineralization in the crown and root areas.

The present findings can be summarized as follows:

1. The profilogram showed an increase in the lower facial height and bimaxillary protrusion with a longer mandible;

2. The dental arch showed a remarkably contracted width in the molar region;

3. The crown widths were larger than the standard values; but

4. The distribution of the degree of dentin mineralization differed little from normal.

The patient has been kept under observation and has undergone periodic examinations to date. It will be important to continue the careful follow-up and to monitor the progress of change to permanent dentition.

\section{Acknowledgments}

The authors are grateful to Dr. G. Yamako, a Public Institute Research staff of the Venture Business Laboratory of Niigata University, for his technical support with the DMB quantitative evaluation system. This study was supported in part by a Grant-in-Aid for Young Scientists (B) (No. 18791548) from the Ministry of Education, Culture, Sports, Science, and Technology of Japan.

\section{References}

1) Sandberg, A.A., Koepf, G.F., Ishihara, T. and Hauschka, T.S.: An XYY human male. Lancet 2: 488-489, 1961.

2) DiGeorge, A.M.: Nelson Textbook of Pediatrics. 15th ed. WB Saunders Co., Philadelphia, 1996, pp. 1629-1633.

3) Gorlin, R.J., Cohen, M.M. and Hennekam, R.C.M.: Syndromes of the Head and Neck. 4th ed. Oxford University Press, New York, 2001, pp.66-67.

4) Varrela, J. and Alvesalo, L.: Effects of the Y chromosome on quantitative growth: an anthropometric study of 47, XYY males. Am J Phys Anthrop 68: 239-245, 1985.

5) Grön, M., Pietilä, K. and Alvesalo, L.: The craniofacial complex in 47, XYY males. Arch Oral Biol 42: 579-586, 1997.

6) Alvesalo, L. and Kari, M.: Sizes of deciduous teeth in 47, XYY males. Am J Hum Genet 29: 486-489, 1977.

7) Townsend, G.: Tooth size in 47, XYY males: evidence for a direct effect of the Y chromosome on growth. Aust Dent J 30: 268-272, 1985.

8) Japanese Society of Pediatric Dentistry: A study on the cephalometric standards of Japanese children. Jpn J Ped Dent 33: 659-696, 1995. (Japanese with English abstract)

9) Moorrees, C.F.: The dentition of the growing child. A longitudinal study of dental development between 3 and 18 years of age. Harvard University Press, Cambridge, 1959.

10) Otsubo, J., Ishikawa, F. and Kuwahara, Y.: A longitudinal study of dental development between 6 to 13 years of age - Growth changes of dentition-. J Jpn Orthod Soc 23: 182-190, 1964. (Japanese with English abstract)

11) Otsubo, J.: A study on the tooth material in Japanese adult of normal occlusion, its relationship to coronal and basal arches. J Jpn Orthod Soc 16: 36-46, 1957. (In Japanese)

12) Japanese Society of Pediatric Dentistry: Research 
concerning the sizes of the primary tooth crown, primary dental arch and condition of primary occlusions of the Japanese. Jpn J Ped Dent 31: 375-388, 1993. (Japanese with English abstract)

13) Augat, P., Gordon, C.L., Lang, T.F., Iida, H. and Genant, H.K.: Accuracy of cortical and trabecular bone measurements with peripheral quantitative computed tomography (pQCT). Phys Med Biol 43: 2873-2883, 1998.

14) Mankani, M.H., Kuznetsov, S.A., Avila, N.A., Kingman, A. and Robey, P.G.: Bone formation in transplants of human bone marrow stromal cells and hydroxyapatite-tricalcium phosphate: prediction with quantitative CT in mice. Radiology 230: 369-376, 2004.

15) Ratcliffe, S.G., Axworthy, D. and Ginsborg, A.: The Edinburgh study of growth and development in children with sex chromosome abnormalities. Birth Defects Orig Artic Ser 15: 243-260, 1979.

16) Shibusawa, F., Fukuda, M., Sakai, H., Sasaki, T. and Imai, Y.: A case of XYY syndrome with velopharyngeal incompetence. J JCPA 29: 175, 2004. (In Japanese) 